\title{
NEUROMOTOR TRANSMISSIBILITY OF HORIZONTAL SEATPAN VIBRATION AND A MATHEMATICAL MODEL
}

\author{
Vinay A.H. Reddy*, Raghu R. Channamallu, Sara E. Wilson \\ Mechanical Engineering, University of Kansas
}

\section{Introduction}

Recent studies of whole body vibration in seated postures have suggested that the neuromotor system may play a role in the etiology of low back disorders ${ }^{1-4}$. A number of researchers have modeled whole body vibration transmission to the low back, spine and head $^{5}$. However, no model to our knowledge has examined the transmission of mechanical vibration to muscle shortening/lengthening, the neuromotor system and reflex muscle activation. In addition, only a few studies have examined biodynamic vibration transmission in the fore-aft (anterior-posterior) direction. In this work, transmission of fore-aft vibration to the spine rotation and erector spinae muscle activation was assessed and a model of the motion was created.

\section{Methods}

Ten healthy young subjects ( 5 male, 5 female, age $24 \pm 3$ years, height $1.6 \pm 0.04 \mathrm{~m}$, weight $69 \pm 4 \mathrm{~kg}$ ) were assessed. Subjects were screened for low back pain and other neuromuscular disorders. The KU-L Human Subjects Committee approved this study and all subjects gave informed consent. A Ling 1512 electro-dynamic shaker was used to create fore-aft vibration. Data from tri-axial accelerometers on the seatpan and attached to the skin at the T10 spinous process, an electrogoniometer across the lumbar spine, electromyography (EMG) on the erector spinae (ES) muscles at L2/L3 was collected during vibration. EMG data was filtered, rectified, integrated and normalized to a maximum obtained prior to vibration exposure. A running average method was used to analyze and obtain a single ensemble average of the processed data for a vibration period. Responses to fore-aft seatpan vibration ( $3 \mathrm{~Hz}$ to $14 \mathrm{~Hz}, 1 \mathrm{~m} / \mathrm{s}^{\wedge} 2 \mathrm{RMS}$ and $2 \mathrm{~m} / \mathrm{s}^{\wedge} 2 \mathrm{RMS}$ ) both with and without a backrest were measured. From the ensemble averages, trunk acceleration transmissibility (seatpan acceleration to T10 accelerometer), vibration transmitted to lumbar rotations (seatpan acceleration to electrogoniometer), vibrationinduced muscle activity (seatpan acceleration to ES EMG) and muscle activity relative to lumbar rotation (electrogoniometer to ES EMG) were calculated.

A lumped parameter model was created with two lumped masses representing head-arm-trunk (HAT) and the pelvis-legs connected with linear and rotational dampers and springs (Figure 1). The parameters for the model were based on weights of the experimental subjects and anthropometric data from literature ${ }^{6}$. Using Lagrangian dynamics, a linearized state-space model was created. This model was used to compare the model to the experimental data. In addition, using Simulink in MATLAB, the vibration experiment was simulated.

\section{Results}

The fore-aft trunk acceleration transmissibility declined with increasing frequency consistent with previous research ${ }^{5}$ and increased with the presence of a backrest. Transmissibility was found to be greater at $2 \mathrm{~m} / \mathrm{s}^{\wedge} 2 \mathrm{RMS}$ compared to $1 \mathrm{~m} / \mathrm{s}^{\wedge} 2 \mathrm{RMS}$. It was observed that the vibration induced lumbar rotations declined with frequency similar 
to trunk acceleration transmissibility but with little change in the presence of a backrest. Examining the relationship between muscle activity and lumbar rotation, the magnitude of muscle activity was found to be mostly linearly related to the magnitude of lumbar rotation, suggesting that lumbar rotation is eliciting the muscle response (Figure 2). The peak muscle activity was delayed relative to peak trunk acceleration, with delays of $390 \mathrm{~ms}$ at $3 \mathrm{~Hz}$ to $43 \mathrm{~ms}$ at $14 \mathrm{~Hz}$, suggesting a transition from voluntary to reflex muscle activation. The model was found to exhibit a similar pattern of fore-aft vibration transmissibility and lumbar rotation as found experimentally. It was also found to exhibit similar patterns of both fore-aft and vertical vibration transmissibility and lumbar rotation as previously reported in the literature ${ }^{5,7}$.

\section{Discussion}

In this work, transmissibility of fore-aft vibration to the low back was found to be consistent with previous literature. Muscle activity in fore-aft vibration was found to correspond to lumbar rotation with delays that suggest a transition from voluntary to reflex-modulated erector spinae muscle response. A mechanical model of trunk dynamics has been created and found to have similar transmissibility and lumbar rotations as were observed experimentally. Future work will be to modify this model to incorporate a Hill type model of muscle dynamics and a model of neuromotor response and to assess the model behavior relative to the muscle activity results found in this foreaft vibration study and previous studies of vertical vibration ${ }^{7}$.

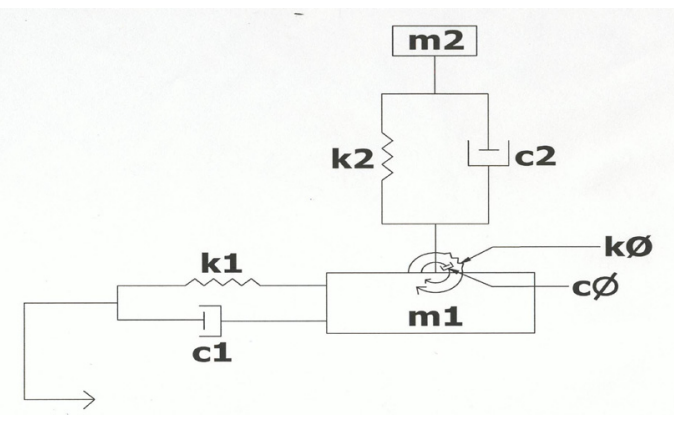

Figure 1 A 2-D model of vibration transmission

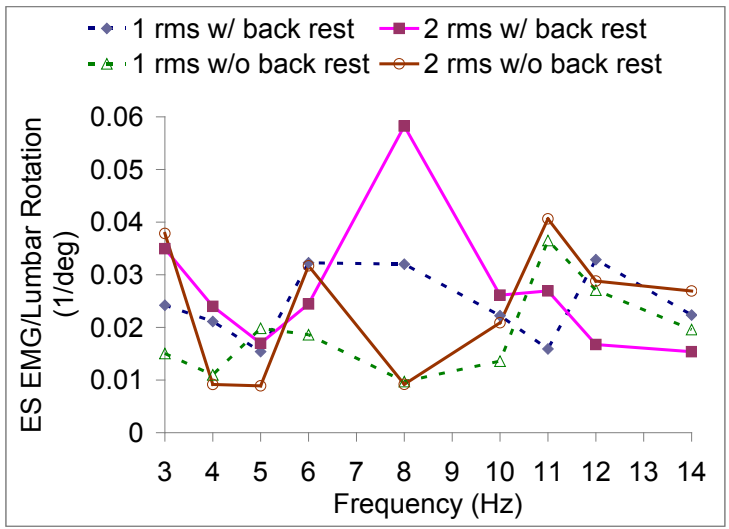

Figure 2 The ratio of muscle activity (fraction of max) to lumbar rotation (deg) was mostly constant with frequency.

\section{References}

1. Wilder, D. G., et al. (1996). Muscular response to sudden load. A tool to evaluate fatigue and rehabilitation. Spine, 21(22), pp. 2628-39.

2. Li, L., et al. (2008). Whole body vibration alters proprioception in the trunk. International Journal of Industrial Engineering, 38(9-10), pp. 792-800.

3. Solomonow, M., et al., (2000). Biexponential recovery model of lumbar viscoelastic laxity and reflexive muscular activity after prolonged cyclic loading. Clinical Biomechanics, 15(3), pp. 167-75.

4. Bluthner, R., et al., (2002). Myoelectric response of back muscles to vertical random whole-body vibration with different magnitudes at different postures. Journal of Sound and Vibration, 253(1), 37-56.

5. Griffin, M. J. (1990). Handbook of Human Vibration, Academic Press Limited, London, England. 6. Winter, D. A. (1990). Biomechanics and Motor Control of Human Movement, Wiley-Interscience, N.Y. 7. Abraham, P. M., and Wilson, S. E. (2006). Whole Body Vibration and Neuromuscular Response. ASME Summer Bioengineering Conference, Amelie Island, Florida. 